\title{
A nomogram to predict prognosis after surgery for young patients with hepatocellular carcinoma
}

\section{Xingchen Li}

Chinese Academy of Medical Sciences and Peking Union Medical College

\section{Xinyu Bi}

China Union Medical University: Chinese Academy of Medical Sciences and Peking Union Medical College

\section{Jianjun Zhao}

China Union Medical University: Chinese Academy of Medical Sciences and Peking Union Medical College

\section{Zhiyu Li}

China Union Medical University: Chinese Academy of Medical Sciences and Peking Union Medical College

\section{Jianguo Zhou}

China Union Medical University: Chinese Academy of Medical Sciences and Peking Union Medical College

\section{Zhen Huang}

China Union Medical University: Chinese Academy of Medical Sciences and Peking Union Medical College

\section{Yefan Zhang}

China Union Medical University: Chinese Academy of Medical Sciences and Peking Union Medical College

\section{Hong Zhao}

China Union Medical University: Chinese Academy of Medical Sciences and Peking Union Medical College

\section{Jianqiang Cai ( $\square$ caijianqiang@cicams.ac.cn )}

China Union Medical University: Chinese Academy of Medical Sciences and Peking Union Medical College

\section{Research}

Keywords: hepatocellular carcinoma, young patients, nomogram, prognosis, prediction model

Posted Date: October 9th, 2020

DOI: https://doi.org/10.21203/rs.3.rs-88877/v1 
License: (9) This work is licensed under a Creative Commons Attribution 4.0 International License. Read Full License

Version of Record: A version of this preprint was published at Translational Cancer Research on April 1st, 2021. See the published version at https://doi.org/10.21037/tcr-20-3411. 


\section{Abstract}

Background Only few studies have been evaluated the clinical characteristics and prognosis of hepatocellular carcinoma in young patients. The purpose of this study is to identify prognostic factors and develop an efficient and practical nomogram to predict cancer-specific survival in young patients with hepatocellular carcinoma.

Methods Four-hundred-and-forty-one young patients with hepatocellular carcinoma who had undergone surgery from 2004-2015 were selected from the Surveillance, Epidemiology, and End Results database. The competing risk model, Lasso and Cox regression were used to screen prognostic factors for cancerspecific survival, and a prognostic nomogram was established using these factors. Thirty-nine young patients with hepatocellular carcinoma from the National Cancer Center, Cancer Hospital, Chinese Academy of Medical Science were used to validate our model. To further evaluate the predictive performance of our model, the concordance index was calculated and the calibration curves were drawn. The clinical usefulness was evaluated by decision curve analysis(DCA). Finally, all patients were grouped by our nomogram. The survival of different risk groups was analyzed using the Kaplan-Meier method, and the differences among survival curves were compared by the log-rank test.

Results The median survival times of the Surveillance, Epidemiology, and End Results training group and the external National Cancer Center validation group were 41 and 52 months, respectively. Histological grade, tumor size, Alpha-fetoprotein, T stage, and M stage were selected as independent factors for cancer-specific survival, and a prognostic nomogram was established. The concordance indices of the training and external validation groups were $0.76(95 \% \mathrm{Cl}, 0.72$ to 0.80$)$ and 0.92 (se=0.085), respectively. The calibration plots showed good agreement. Decision curve analysis revealed that our nomogram resulted in a better clinical net benefit than the AJCC 7th edition and Barcelona Clinic Liver Cancer staging systems. Patients were divided into two risk groups according to the cut-off value of 125 of the total points from our nomogram. Kaplan-Meier plots for cancer-specific survival were performed using the logrank test, the $p$-value of which was $<0.001$.

Conclusions The practical nomogram resulted in a more-accurate prognostic prediction for young hepatocellular carcinoma patients after curative liver resection.

\section{Introduction}

Liver cancer is the fourth most common cause of cancer-related death, with the sixth-highest incidence rate worldwide (1). Hepatocellular carcinoma (HCC) accounts for the majority of primary liver cancer; however, the efficacy of therapies for HCC is poor. Although HCC is typically diagnosed in middle-aged and elderly populations, the peak incidence age differs substantially according to geographical regions and etiology. Hepatitis B virus (HBV)-associated HCC patients in East Asia and Africa are generally younger than those with hepatitis $\mathrm{C}$ virus (HCV)-associated HCC in Western countries (2). Moreover, in high-risk areas of East Asia, HCC is often diagnosed in individuals younger than 40 years of age, which is rare in European countries (3). Compared with middle-aged and elderly individuals, the liver cirrhosis rate of 
young patients is lower, and the liver function of these patients is better; however, HBV infection rates and serum alpha-fetoprotein (AFP) levels are higher among younger patients, and they generally have large tumor diameters, worse tumor differentiation, and stronger invasion $(4,5)$. HCC patients often have a threestep development process of hepatitis-cirrhosis-liver cancer, but the process of cirrhosis in young patients is not apparent; therefore, researchers hypothesized that the tumorigenesis mechanisms of HCC are dissimilar at different ages (4). However, there are few studies on the risk factors for HCC in young patients. Several studies have found that the prognosis of HCC in young patients is better than that in older patients and that there is no significant difference in overall survival (OS) and disease-free survival (DFS) between the two age groups (5-7), while other studies have shown that the prognosis of young patients is worse (8-10). The disparities in these studies may be due to individual differences in the data of the patients; there is no strict matching between the clinical baseline data of the two groups of patients. In general, the clinicopathological characteristics of young patients with liver cancer are specific, and the prognosis is controversial.

The Surveillance, Epidemiology, and End Results (SEER) program is one of the largest and most representative cancer registration databases in North America and provides extensive data for different types of malignancies. It serves as an exhaustive source of information on malignancies in the United States, which affects approximately $35 \%$ of the U.S population (11).

Nomograms help to quantify various factors and use them for constructing a model to predict the outcome (12). This model could be used to predict specific outcomes for any individual patient. The one in ten rule is an empirical rule for how many predictor parameters can be estimated from data when conducting regression analysis while maintaining the risk of overfitting low in statistics. The rule states that one predictive variable can be studied for every ten events $(13,14)$; due to the low incidence of HCC in young patients, it is not statistically effective to build the model with single-center data. In this study, the clinicopathological features and survival data of patients with HCC were obtained from the SEER database, which was used as a training cohort for constructing a nomogram to predict CSS. The prognostic evaluation models (PEM) constructed with the nomogram were then validated with an external cohort of HCC patients. We present the following article in accordance with the TRIPOD reporting.

\section{Methods}

\section{Ethics statement}

Ethical approval was obtained from the Ethics Committee of the Cancer Hospital, Chinese Academy of Medical Sciences. All external validation group patients had given written informed consent. The study complied with the Declaration of Helsinki.

\section{Data acquisition and the process of patient selection in the training group}

The data were obtained from the SEER database (SEER 18 Regs Custom Data (with additional treatment fields), Nov 2018 sub (1973-2016 varying)) using the SEER*Stat software (version 8.3.6). In the SEER 
database, the surgical information about the primary site was only available after 1988. Generally, Collaborative Stage (CS) information must be acquired to obtain more detailed information on the American Joint Committee on Cancer (AJCC) staging. However, this information was only available between 2004 and 2015; hence, we included HCC patients from 2004 to 2015.

Inclusion criteria include (1) patients diagnosed with HCC from 2004 to 2015 (histologic type ICD-O$3=8170-8175$ ), (2) a maximum age of 40 years, and (3) patients with available follow-up data. Exclusion criteria were listed as following: (1) patients with a history of other malignancies, (2) the patient did not undergo surgical treatment, and (3) the diagnosis of the disease was not based on the results of pathology or cytology. The selection process for enrolling patients is shown in Figure 1.

\section{External validation group}

Our study's independent external validation population was drawn from 39 consecutive young HCC patients from March 2012 to November 2017, who were treated with hepatic surgery at the National Cancer Center (NCC) Cancer Hospital, Chinese Academy of Medical Science. The inclusion and exclusion criteria were the same as for the SEER training group. The follow-up information was obtained via telephone, e-mail, letter, or outpatient service. Furthermore, similar variable information as for the SEER training group was included. All external validation group patients were staged with the following two major staging systems: the AJCC tumor-nodes-metastasis (TNM) staging system (7th edition 2010) and the Barcelona Clinic Liver Cancer (BCLC) staging system.

\section{Definition of primary endpoint and staging system}

In the statistical analysis process, we mainly applied the following two outcome indicators: cancer-specific survival (CSS) and survival time. CSS was determined as the time interval from the diagnosis date until the date of tumor-related death. If a patient died due to a non-tumor cause, we classified it as another type of non-censored data. Patients who survived at the end of follow-up were classified as censored. In the SEER database, when the survival time is less than one month, it is defined as zero. According to standard epidemiological conventions, a survival of 0.5 months was assigned to those patients. Hepatocellular carcinoma was staged using the $7^{\text {th }}$ edition of the AJCC TNM staging system. Only the patients from 2010 to 2015 in the original SEER data had information regarding the 7th edition AJCC staging system. For other patients, the fields of CS Extension $₫$ CS Lymph Nodes $₫$ CS Mets at DX, and CS tumor size were used to determine the 7th edition AJCC staging.

\section{Statistical analysis}

First, risk factors related to CSS were screened based on a competing risk model. Gray's test was used to compare the cumulative incidence of death due to cancer and death due to other reasons, such as competing events (15). Factors with a $P$ value of less than 0.2 entered the subsequent univariate Cox analysis. To further in-depth analyze the variables related to CSS, patients who did not die from hepatocellular carcinoma were excluded. Second, to minimize the possibility of overfitting of our model, 
variables with a $P$ value less than 0.05 in the univariate Cox analysis were included in the Lasso penalized cox model to filter the variables further. LASSO regression could set the coefficients of many irrelevant features exactly to zero based on the regulation weight $\lambda$. Optimal values of $\lambda$ were determined by 10 -fold cross-validation(16) . Finally, the results of the LASSO regression were included in the multivariate cox regression analysis, and the final CSS-related variables were selected based on the P-value of less than 0.05. All p-values were reported to 3 decimal places. Subsequently, a nomogram of young liver cancer patients using these factors was constructed to predict 1-, 2-, and 3-year CSS.

The discriminative ability was evaluated using the c-index and receiver operating characteristics (ROC) curves of different times. The nomogram is further assessed for its predictive accuracy using calibration curves. The calibration curve can be used to observe the agreement between the predicted value and the actual value. In the calibration curve analysis, the prediction error was assessed using the nonparametric bootstrap procedure with 500 bootstrap replicates to minimize bias.

The decision curve analysis (DCA) was plotted to examine the usefulness and benefit of our nomogram. Our nomograms were subjected to 1000 bootstrap resamples for internal validation. An independent external validation group from NCC was used to validate our model. The total score of each patient in the validation set was calculated according to the generated nomogram. Then, cox regression was performed using the total score as a factor. The C-index, calibration curve, and clinical decision curve were derived based on the cox regression in the validation group. The cut-off value of total score were analyzed using the X-tile program to distinguish the differences in young HCC patients' survival. The X-tile program is used to find the optimal value of continuous variables, and the program's application has been reported in numerous studies(17). We then stratified patients into different risk groups according to the cut-off value for the total points. The Survival curves of the different risk groups were plotted by the Kaplan-Meier method, and the log-rank test was used to determine significance.

Statistical analysis was performed using R (version 3.6.2) software. Statistical significance was set as above.

\section{Results}

\section{Characteristics of training and validation groups}

A total of 441 young HCC patients from 2004 to 2015 were included in our training group from the SEER database, of whom 284 cases (6.6\%) were male. The median survival time of the training group was 41.00 (17.00-86.00) months. Among them, the cause of death in 20 patients was non-tumor or unknown; therefore, in the process of screening for CSS risk factors, they were deleted. The validation group included 39 patients from the NCC who underwent surgical treatment in our hospital from March 2012 to November 2017. The last follow-up time was March 2020. Seven patients died, and the cause of death was HCC. The clinical and pathological features of the training and validation groups are listed in Table 1.

Table 1. Clinical and pathological features of the training and validation groups 


\begin{tabular}{|c|c|c|}
\hline Variable & $\begin{array}{l}\text { SEER training group } \\
\text { number }(\%)\end{array}$ & $\begin{array}{c}\text { CICAMS validation group } \\
\text { number }(\%)\end{array}$ \\
\hline Number of patients & 441 & 39 \\
\hline \multicolumn{3}{|l|}{ Sex } \\
\hline Male & $284(64.40 \%)$ & 37 (94.8\%) \\
\hline Female & $157(35.60 \%)$ & $2(5.1 \%)$ \\
\hline $\mathrm{Age}^{\mathrm{a}}$ & $32.00(21.00-38.00)$ & $35.00(31.50-39.00)$ \\
\hline \multicolumn{3}{|l|}{ Race $^{\text {b }}$} \\
\hline White & $265(60.09 \%)$ & - \\
\hline Black & $50(11.34 \%)$ & - \\
\hline Other & $122(27.66 \%)$ & $39 \square 100 \% \square$ \\
\hline Unknown & $4(0.91 \%)$ & - \\
\hline \multicolumn{3}{|l|}{ Insurance recode } \\
\hline Insured and any medicaid & $288(65.31 \%)$ & $35(89.74 \%)$ \\
\hline Uninsured & $8(1.81 \%)$ & $4(10.26 \%)$ \\
\hline Unknown & $145(32.88 \%)$ & 0 \\
\hline \multicolumn{3}{|l|}{ Marital status } \\
\hline Unmarried $^{\mathrm{c}}$ & $169(38.32 \%)$ & $14(35.90 \%)$ \\
\hline Marrierd & $260(58.96 \%)$ & $25(64.10 \%)$ \\
\hline Unknown & $12(2.72 \%)$ & $0(38.32 \%)$ \\
\hline \multicolumn{3}{|l|}{ Grade } \\
\hline Grades I+II & $111(25.17 \%)$ & $10(25.64 \%)$ \\
\hline Grades III+IV & $252(57.14 \%)$ & $15(38.46 \%)$ \\
\hline Unknown & $111(25.17 \%)$ & $14(35.89 \%)$ \\
\hline \multicolumn{3}{|l|}{ AJCC stage 7th } \\
\hline I & $190(43.08 \%)$ & $30(76.9 \%)$ \\
\hline II & $93(21.09 \%)$ & $3(7.6 \%)$ \\
\hline III & $74(16.78 \%)$ & $5(12.8 \%)$ \\
\hline IV & $62(14.06 \%)$ & $0(0 \%)$ \\
\hline Unknown & 22 (4.99\%) & $1(2.5 \%)$ \\
\hline \multicolumn{3}{|l|}{ T stage } \\
\hline $\mathrm{T} 1$ & $210(47.62 \%)$ & $30(76.92 \%)$ \\
\hline $\mathrm{T} 2$ & $106(24.04 \%)$ & $3(7.69 \%)$ \\
\hline T3 & 89 (20.18\%) & $5(12.82 \%)$ \\
\hline $\mathrm{T} 4$ & $21(4.76 \%)$ & $0(0 \%)$ \\
\hline
\end{tabular}


Tx

N stage

NO

N1

$\mathrm{Nx}$

\section{M stage}

M0

M1

$\mathrm{Mx}$

BCLC

0

A

B

C

Surgery to the liver-detail

Local tumor destruction

Wedge or segmental resection

Lobectomy

Extended lobectomy

Hepatectomy,NOS

Surgery, NOS

\section{Surgery to LN}

No

Yes

Unknown

AFP

Positive/elevated

Negative/normal

Unknown

\section{Fibrosis score}

0-4

5-6

Unknown

\section{Tumor size group(mm)}

0-20

21-50

$15(3.40 \%)$

$1(2.56 \%)$

388 (87.98\%)

39 (100.00\%)

$40(9.07 \%)$

$0(0 \%)$

$13(2.95 \%)$

$0(0 \%)$

402 (91.16\%)

39 (100\%)

35 (7.94\%)

$0(0 \%)$

$4(0.91 \%)$

$0(0 \%)$

$3(7.6 \%)$

$25(64.1 \%)$

$11(28.2 \%)$

$0(0 \%)$

32 (7.26\%)

$132(29.93 \%)$

$128(29.02 \%)$

$34(7.71 \%)$

107 (24.26\%)

$8(1.82 \%)$

$302(68.48 \%)$

136 (30.84\%)

$3(0.68 \%)$

196 (44.44\%)

$144(32.65 \%)$

101 (22.90\%)

71 (16.10\%)

43 (9.75\%)

327 (74.15\%)

55 (12.47\%)

114 (25.85\%)
$0(0 \%)$ $26(66.6 \%)$ $4(10.2 \%)$ 26(66.6\%) $2(5.1 \%)$

7(17.9\%)

$35(89.7 \%)$

$4(10.2 \%)$

$0(0 \%)$

25(64.1\%)

13(33.3\%)

$1(2.5 \%)$

$13(34.21 \%)$

15 (39.47\%)

$10(26.32 \%)$

1 (2.56\%)

21 (53.85\%) 
51-100

$\geq 101$

Unknown

Overall survival

Alive

Dead

Cancer-specific survival

Alive

Death due to cancer

Death due to other reasons

Survival months ${ }^{a}$
109 (24.72\%)

$140(31.75 \%)$

$23(5.22 \%)$

$266(60.32 \%)$

175 (39.68\%)

$266(60.32 \%)$

155 (35.15\%)

$20(4.54 \%)$

41.00 (17.00-86.00)
$10(25.64 \%)$

$6(15.38 \%)$

$1(2.56 \%)$
$32(82.05 \%)$

7 (17.95\%)

$32(82.05 \%)$

7 (17.95\%)

$0(0 \%)$

a:median (Q1-Q3)

b:includes American Indian/AK Native, Asian/Pacific Islander

c:divorced/separated/single (never married)/unmarried or domestic partner/widowed

\section{Prognostic factors related to CSS for young HCC patients}

In Gray's test, marital status, histological grade, size group, AFP, T stage, N stage, and M stage were associated with CSS. The Cumulative incidence function (CIF) of young HCC patients with risk factors with a P-value $<0.2$ for CSS is displayed in Figure 2. After deleting the data of patients who died from nontumor causes, the data of 421 patients entered the follow-up analysis. After primary Cox univariate analysis filtration, the remaining six variables were included in the Lasso regression. We applied 10x crossvalidation to find the minimum of partial likelihood deviance which could make our model simpler. A simplified model helped to avoid overfitting as much as possible. Finally, the selected variables when the partial likelihood deviance was minimal (lambda=-4.44) were grade, size group, AFP, T stage, and M stage. Additional details of the prognostic factor selection using the LASSO regression model are disclosed in Figure 3A-B. In the multivariate Cox analysis, these five variables remained independently related to CSS. The variables screening process related to CSS details is outlined in Table 2. Collinearity diagnostics were examined for the potential presence of collinearity between the independent variables, and Variance inflation factors (VIF) $\leq 5$.

Table 2. Variable screening process related to CSS 


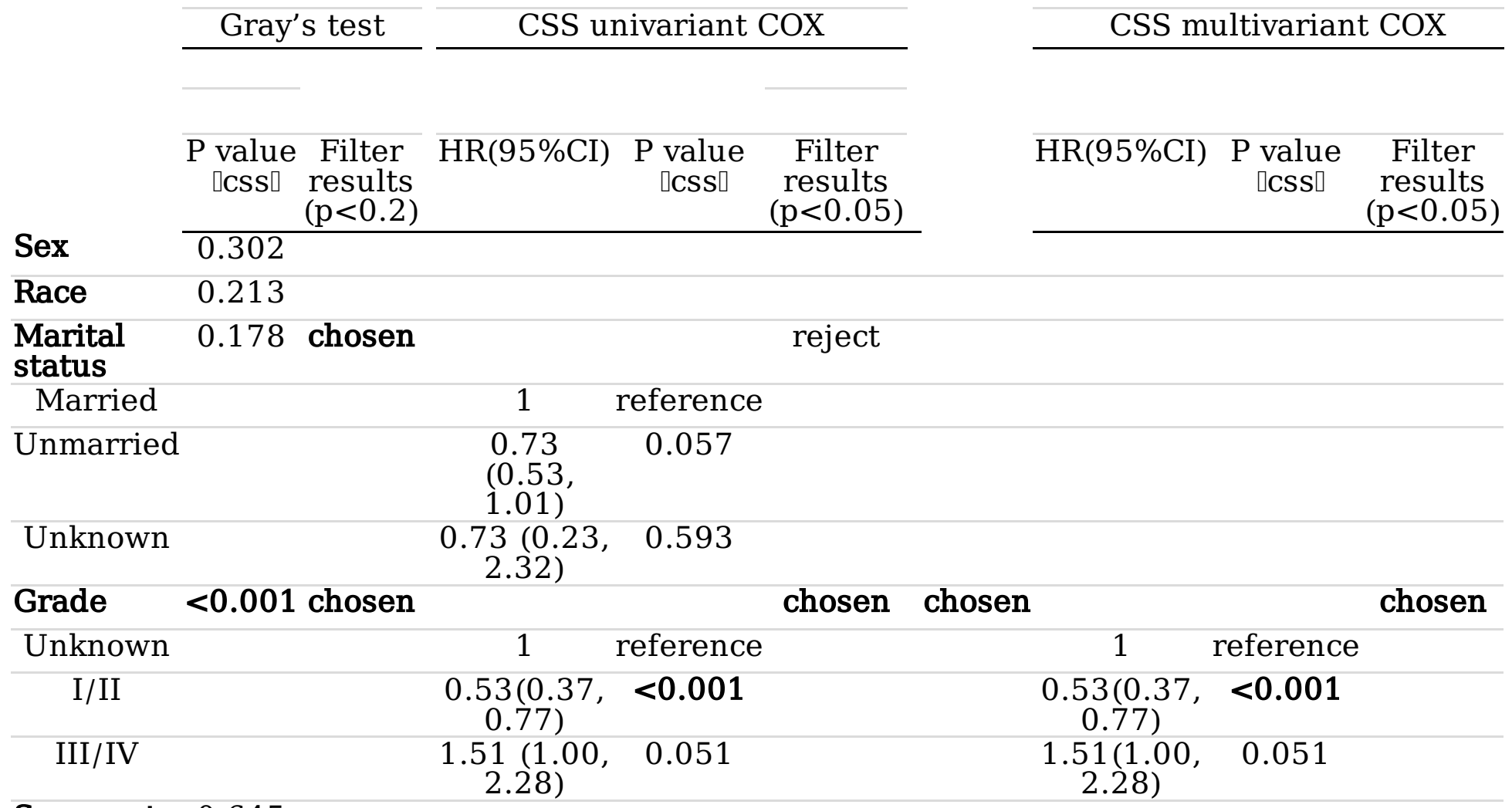

Surgery to 0.645

LN

No

Yes

unknown

Size group $<0.001$ chosen

chosen chosen

chosen

0-20

21-50

51-100

$101+$

unknown

AFP $<0.001$ chosen

Positive

Negative

unknown

Fibrosis

Score

Fibrosis

score 5-6

Fibrosis

score $0-4$

unknown

Insurance

T stage $<0.001$ chosen

0.795
1 reference

$1.92(1.17, \quad 0.001$ $3.14)$

$5.05(3.21,<0.001$ 7.97)

$5.19(3.25,<0.001$

$8.28)$

$1.96(0.86,0.108$

4.43)

1 reference

chosen chosen

$1.51(0.64,0.346$

$3.55)$

$3.46(1.55, \quad 0.002$ $7.71)$

$5.46(2.52,<0.001$ $11.86)$

$3.50(1.30, \quad 0.013$

9.39)

1 reference
$0.45(0.31,<0.001$ $0.66)$
$0.44(0.29,<0.001$
$0.68)$
$0.45(0.31,<0.01$
$0.66)$
$0.44(0.29, \quad<0.01$
0.68 )

chosen 
$\mathrm{T} 1 / 0$

$\mathrm{T} 2$

T3

$\mathrm{T} 4$

TX

N stage $\quad 0.010$ chosen

NO

N1

NX

M stage $<0.001$ chosen

M0

M1

MX

$4.85(3.23,<0.001$

7.27)

$8.67(4.75,<0.001$

15.85)

$1.55(0.55, \quad 0.402$

4.34)

chosen reject

1 reference

$1.76(1.11, \quad 0.017$

2.79)

$2.00(0.93, \quad 0.075$

4.29)

chosen chosen

chosen

1 reference

$3.49(2.33,<0.001$

$5.24)$

$0.67(0.09,0.693$

4.81)

\section{Construction and validation of the nomogram}

Five variables, grade, size group, AFP, T stage, and M stage, were associated with CSS and were used to construct a nomogram, as shown in Figure 4. The C-index of nomogram in the SEER training group was $0.76(95 \% \mathrm{Cl}, 0.72$ to 0.80$)$. Time-dependent ROC curves of CSS in the SEER group showed that the AUC at 1,2 and 3 years was $0.81,0.77$, and 0.76 , respectively. The C-index of the $7^{\text {th }}$ AJCC system was 0.68 ( $95 \%$ $\mathrm{Cl}, 0.63$ to 0.72 ). The AUC for $1-, 2$-, and 3 - years of the $7^{\text {th }}$ edition AJCC staging system was $0.70,0.67$, and 0.69 , respectively. The C-index of NCC validation group was $0.92(95 \% \mathrm{Cl}, 0.84$ to 1.00$)$.

Time-dependent ROC curves showed that the AUC at 1, 2 and 3 years was $0.95,0.91$, and 0.85 , respectively. The C-index of the AJCC and BCLC staging systems were only 0.80 ( $95 \% \mathrm{Cl}, 0.66$ to 0.95$)$ and $0.61(95 \% \mathrm{Cl}, 0.42$ to 0.80$)$, respectively.

The calibration curves illustrated an optimal agreement in the probability of 1-and 3-year CSS between the prediction by nomogram and actual observation in both in the SEER training and NCC validation groups (see Figure 5 for details). Furthermore, the DCA was plotted and our nomogram showed better net benefits with a wider range of threshold probability than either the 7th edition AJCC or BCLC staging systems in the training and validation groups (see Figure 6 for details).

\section{Risk stratification according to the nomogram}

According to the nomogram model, the total score of each training group patient were calculated. The total points ranged from 0 to 244. The best cut-off value of total score was 125 and the patients could be divided into low-risk (score $\leq 125)$ and high-risk group (score冈125). Kaplan-Meier survival curves of the 
training and validation groups were generated. The log-rank test was used in analysis and the p-value $<0.001$ (see further details in Figure 7).

\section{Discussion}

This study included the largest cohort of young patients with HCC to date as we explored the characteristic of this tumor using the SEER database. The clinicopathological characteristics and prognosis of this population are significantly different from those of middle-aged and elderly patients with liver cancer. Our research focused on identifying prognostic risks and clinicopathological factors, constructing PEM, and validating with an external validation cohort. We identified grade, size group, AFP, $\mathrm{T}$ stage, and $\mathrm{M}$ stage as independent prognostic factors for HCC in young patients. Moreover, a nomogram we constructed that contained the risk factors associated with the prognosis of HCC to predict the CSS of young patients. The calibration curves for 1-, 2-, and 3-year CSS closely matched the ideal 45 degree line, and the c-indices of the training and external validation groups were 0.76 and 0.92 , respectively, which was significantly higher than that of the conventional staging systems-the $7^{\text {th }}$ edition AJCC and BCLC. These comprehensive analysis designs for screening prognostic factors contributed to the results with high credibility and good predictive effect.

In recent years, the number of young people with $\mathrm{HCC}$ has increased (18). There are no specific clinical manifestations of HCC in young patients, but the tumor typically grows insidious and fast. Young people seldom have regular physical examinations, and they often do not pay attention to it and are prone to delay the disease. Compared with middle-aged and elderly people, young people often show high AFP levels, large tumor diameter, low tumor differentiation, and high HBV infection rates, but a low degree of cirrhosis $(19,20)$. A study demonstrated that a younger group of patients showed better liver function and had higher AFP levels than an older group; these outcomes are consistent with other previous reports. In addition, younger patients tend to have larger tumors than older patients (6). Overall, the prognosis of young patients with HCC is controversial (5-10). Several researchers have reported that young HCC patients tend to have poor prognoses, compared to middle-aged or older patients. This is most likely because young $\mathrm{HCC}$ patients are often diagnosed with advanced stages, as indicated by their advanced TNM stages and high serum AFP levels. In addition, certain patients with HCC may have developed the disease from HBV infection; the proportion is significantly higher than that in elderly patients (19). HBV can develop in non-cirrhotic livers and is also an underlying cause of HCC in young patients. This may be one of the reasons why there is a lesser degree of liver cirrhosis in young patients, and the prognosis of young patients with HCC is better. Other reports have indicated that there is no significant difference in OS and DFS between younger and older patients $(5,6)$.

As a practical tool in clinical practice, the nomogram is more accurate and individualized in predicting the survival and prognosis of patients than traditional neoplasm staging criteria and has increasingly attracted attention from clinicians. Since the results of the regression equation can be visualized by the nomogram, it has been used to predict the survival and recurrence of various tumors, such as breast cancer (21) and lung cancer (22). A nomogram model for survival or postoperative recurrence of liver 
cancer has been reported (23-26); the survival of patients with HCC is affected by numerous factors, but most of the predictive factors in these studies only involve the characteristics of tumors, and many are single-center studies without external verification. Since most of the patients with HCC are middle-aged and elderly, the previous prediction model is more suitable for these patients. Research has reported nomograms built on multivariate analyses, which showed that one of the independent predictors for HCC was cirrhosis (27), which is typically more serious in elderly patients compared to young HCC patients. We hope to establish a more comprehensive and practical nomogram model for the specific group of HCC in young patients, including tumor characteristics, pathological characteristics, and other factors. The incidence of HCC in young people is relatively low, which makes it more important to establish a prediction model by using public databases, to enlarge the advantage of sample size. In addition, the application of the nomogram model must be established on the premise that the model has been verified to ensure the reliability and accuracy of the prediction effect. Moreover, in the present study, the PEM developed were both internally and externally validated; the c-indices of the training and external validation groups were 0.76 and 0.92 , respectively, and the calibration curves for the probability of survival showed good agreement.

This study had several limitations, including the lack of reports on hepatic virus infections, such as $\mathrm{HBV} / \mathrm{HCV}$, surgical procedures, and liver function, which was due to that fact that they were not completely recorded in the SEER database. Therefore, the optimal management of HCC in young patients could not be studied. In addition, the retrospective study design has several inherent and inevitable biases, which might be resolved by confirming the study results with large-scale prospective multicenter studies with a more comprehensive analysis of the risk factors. Regardless of these limitations, our study remains the largest population-based HCC study to date and provides a specific predictive model for HCC prognosis in young patients. Further validation with a larger HCC cohort is needed in the future.

\section{Conclusion}

Grade, tumor size, Alpha-fetoprotein (AFP), T stage, and M stage were independent prognostic factors associated with CSS in young HCC patients. The nomogram could be used in clinical practice to accurately predict the prognosis of young HCC patients.

\section{Declarations}

\section{Funding}

This work was supported by the National Capital Health Research and Development of Special (No. 20181-4021) and the National Science and Technology Major Project (No. 2018ZX10723204).

\section{Availability of data and materials}

The data that support the findings of this study are available from the corresponding author upon reasonable request. 


\section{Acknowledgments}

None

\section{Authors' contributions}

Conception and design: JC, HZ. Administrative support: JC, HZ. Provision of study materials of patients: $\mathrm{XB}, \mathrm{JZ}, \mathrm{ZL}$. Collection and assembly of data: JZ, ZH, YZ. Data analysis and interpretation: XL. Manuscript writing: All authors. Final approval of manuscript: All authors.

\section{Ethics approval and consent to participate}

The authors are accountable for all aspects of the work in ensuring that questions related to the accuracy or integrity of any part of the work are appropriately investigated and resolved. The research was in compliance with the Declaration of Helsinki. Ethical approval was obtained from the Institutional Review Board of the Cancer Hospital, Chinese Academy of Medical Sciences (ID: NCC2019C-016), and the necessity for informed consent was waived.

\section{Consent for publication}

The consent for publication of the individual patient data from the patient has been obtained by Cancer Hospital, Chinese Academy of Medical Sciences.

\section{Competing interests}

The authors declare that they have no competing interests.

\section{References}

1. International Agency for Research on Cancer WHO. Cancer today (https://gco.iarc.fr/today/home).

2. Klein WM, Molmenti EP, Colombani PM, et al. Primary liver carcinoma arising in people younger than 30 years. Am J Clin Pathol 2005;124:512-8.

3. Bosch FX, Ribes J, Diaz M, et al. Primary liver cancer: worldwide incidence and trends. Gastroenterology 2004;127:S5-S16.

4. Namieno T, Kawata A, Sato N, et al. Age-related, different clinicopathologic features of hepatocellular carcinoma patients. Ann Surg 1995;221:308-14.

5. Lee CR, Lim JH, Kim SH, et al. A comparative analysis of hepatocellular carcinoma after hepatic resection in young versus elderly patients. J Gastrointest Surg 2012;16:1736-43.

6. Lee JS, Kim JM, Lee S, et al. The prognosis in cases of hepatocellular carcinoma after hepatectomy: young patients versus older patients. Korean J Hepatobiliary Pancreat Surg 2015;19:154-60.

7. Falkson G, Cnaan A, Schutt AJ, et al. Prognostic factors for survival in hepatocellular carcinoma. Cancer Res 1988;48:7314-8. 
8. $\mathrm{Ni} \mathrm{YH}$, Chang MH, Hsu HY, et al. Hepatocellular carcinoma in childhood. Clinical manifestations and prognosis. Cancer 1991;68:1737-41.

9. Cho SJ, Yoon JH, Hwang SS, et al. Do young hepatocellular carcinoma patients with relatively good liver function have poorer outcomes than elderly patients? J Gastroenterol Hepatol 2007;22:1226-31.

10. Chen $\mathrm{CH}$, Chang TT, Cheng KS, et al. Do young hepatocellular carcinoma patients have worse prognosis? The paradox of age as a prognostic factor in the survival of hepatocellular carcinoma patients. Liver Int 2006;26:766-73.

11. Software SID-SD. https://seer.cancer.gov/data/index.html. SEER 2019.

12. Kattan MW, Scardino PT. Evidence for the usefulness of nomograms. Nat Clin Pract Urol 2007;4:6389.

13. Harrell FE, Jr., Lee KL, Mark DB. Multivariable prognostic models: issues in developing models, evaluating assumptions and adequacy, and measuring and reducing errors. Stat Med 1996;15:361-87.

14. Peduzzi P, Concato J, Kemper E, et al. A simulation study of the number of events per variable in logistic regression analysis. J Clin Epidemiol 1996;49:1373-9.

15. Gray R. A Class of \$K\$-Sample Tests for Comparing the Cumulative Incidence of a Competing Risk. The Annals of Statistics 1988;16.

16. Tibshirani R. Regression shrinkage selection via the LASSO. Journal of the Royal Statistical Society Series B 2011;73:273-82.

17. Camp RL, Dolled-Filhart M, Rimm DL. X-tile: a new bio-informatics tool for biomarker assessment and outcome-based cut-point optimization. Clin Cancer Res 2004;10:7252-9.

18. Zhang $\mathrm{S}$, Zheng $\mathrm{R}$, Zeng $\mathrm{H}$, et al. [The incidence differences among sex, geographical areas and mean age of diagnosis for liver cancer in China, 1989-2008]. Zhonghua Yu Fang Yi Xue Za Zhi 2014;48:35560.

19. Yamazaki Y, Kakizaki S, Sohara N, et al. Hepatocellular carcinoma in young adults: the clinical characteristics, prognosis, and findings of a patient survival analysis. Dig Dis Sci 2007;52:1103-7.

20. Kim JH, Choi MS, Lee H, et al. Clinical features and prognosis of hepatocellular carcinoma in young patients from a hepatitis B-endemic area. J Gastroenterol Hepatol 2006;21:588-94.

21. Katz A, Smith BL, Golshan M, et al. Nomogram for the prediction of having four or more involved nodes for sentinel lymph node-positive breast cancer. J Clin Oncol 2008;26:2093-8.

22. Liang $W$, Zhang $L$, Jiang $G$, et al. Development and validation of a nomogram for predicting survival in patients with resected non-small-cell lung cancer. J Clin Oncol 2015;33:861-9.

23. Li J, Liu Y, Yan Z, et al. A nomogram predicting pulmonary metastasis of hepatocellular carcinoma following partial hepatectomy. Br J Cancer 2014;110:1110-7.

24. Shim JH, Jun MJ, Han S, et al. Prognostic nomograms for prediction of recurrence and survival after curative liver resection for hepatocellular carcinoma. Ann Surg 2015;261:939-46.

25. Shen J, He L, Li C, et al. Prognostic nomograms for patients with resectable hepatocelluar carcinoma incorporating systemic inflammation and tumor characteristics. Oncotarget 2016;7:80783-93. 
26. Feng LH, Dong H, Lau WY, et al. Novel microvascular invasion-based prognostic nomograms to predict survival outcomes in patients after R0 resection for hepatocellular carcinoma. $\mathrm{J}$ Cancer Res Clin Oncol 2017;143:293-303.

27. Torzilli G, Donadon M, Belghiti J, et al. Predicting Individual Survival After Hepatectomy for Hepatocellular Carcinoma: a Novel Nomogram from the "HCC East \& West Study Group". J Gastrointest Surg 2016;20:1154-62.

\section{Figures}

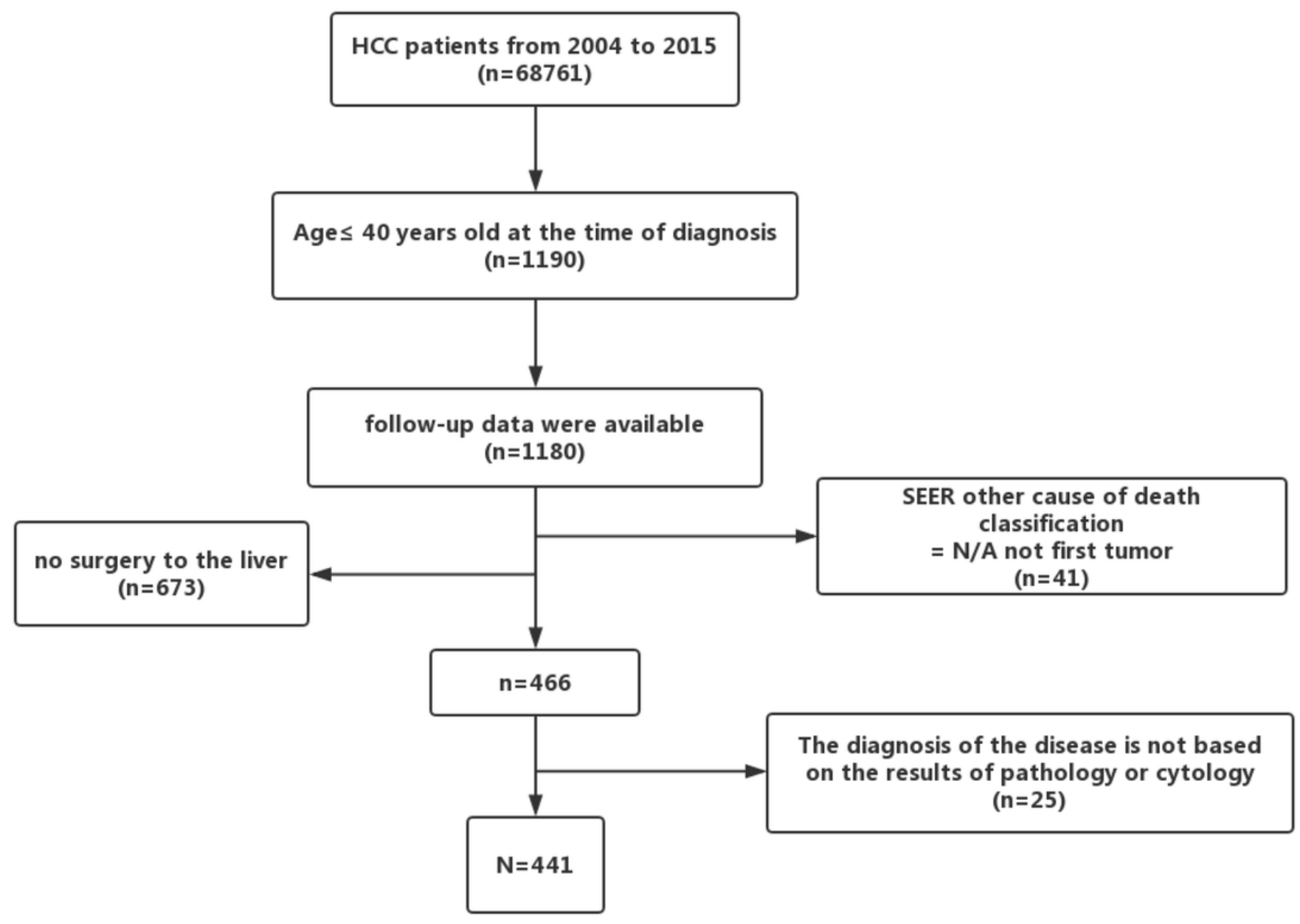

\section{Figure 1}

Flow chart of patients selection in the training group from the SEER database 

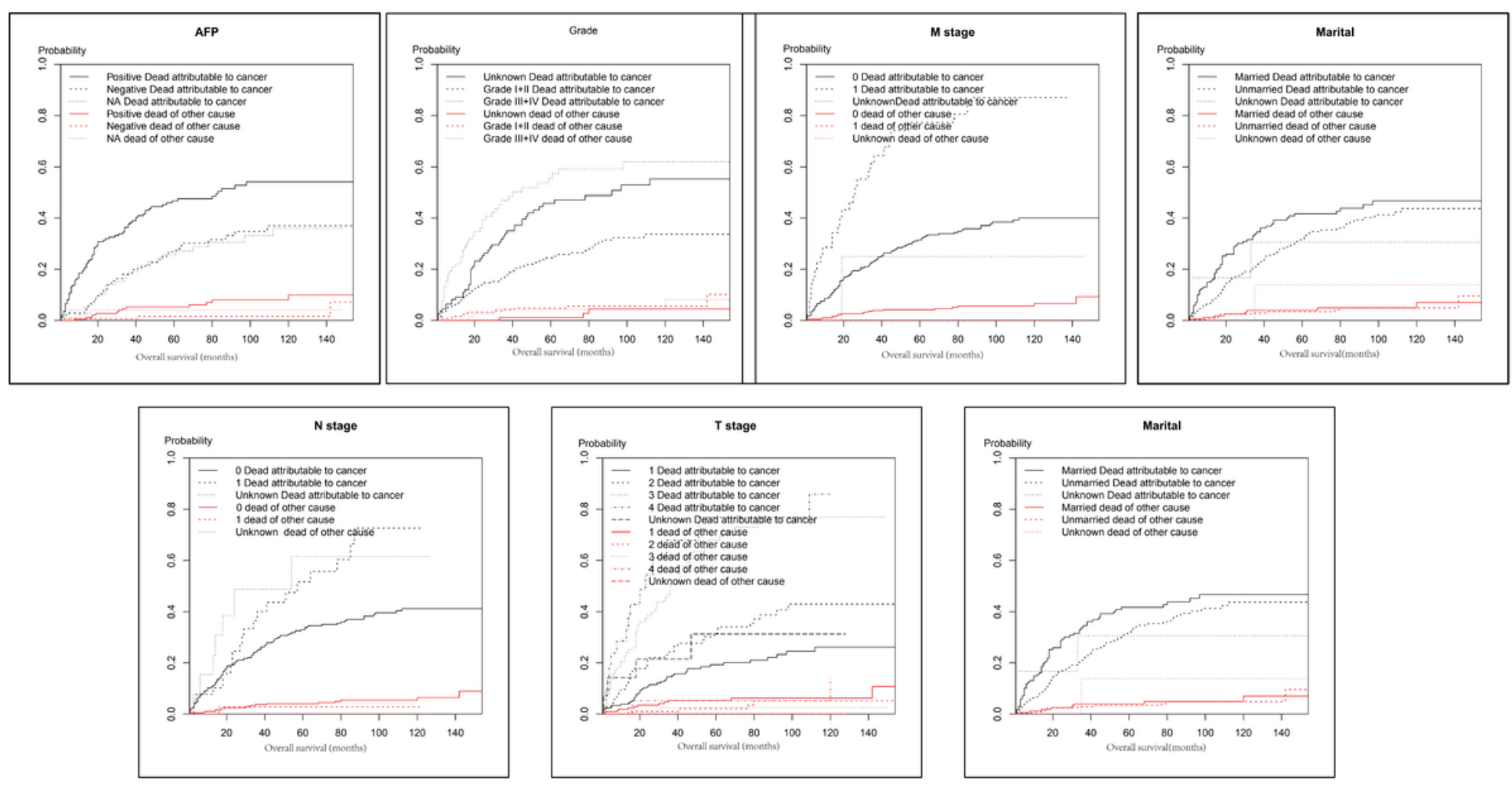

\section{Figure 2}

Cumulative incidence function (CIF) of SEER training patients with risk variables for cancer-specific death
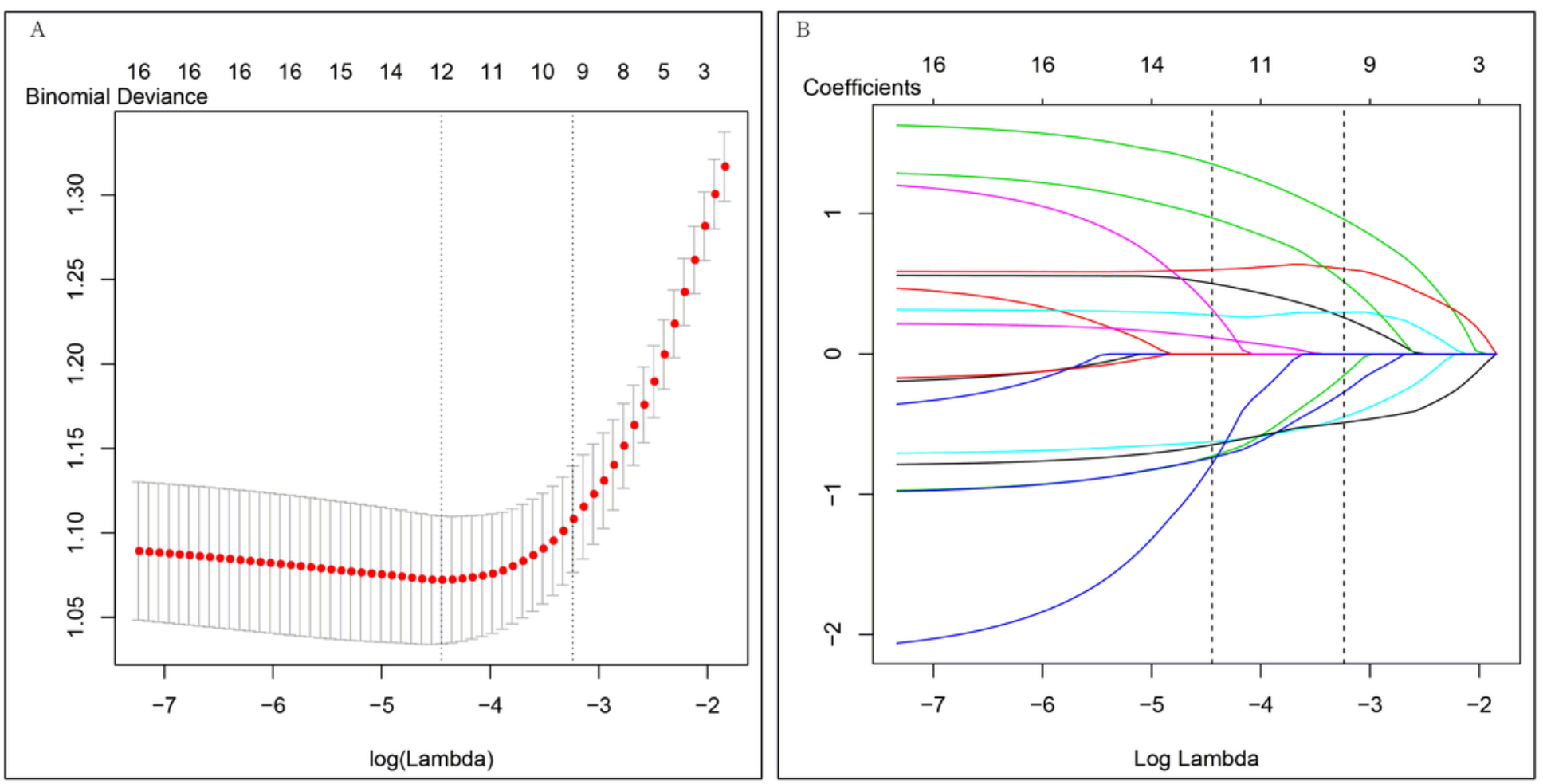

Figure 3 
Prognostic factor selection using LASSO regression model. 3A: Lasso regression search for the optimal coefficient when the Lambda was -4.44 3B:10x cross-validation was applied for searching the Lambda when partial likelihood deviance was the least

Points

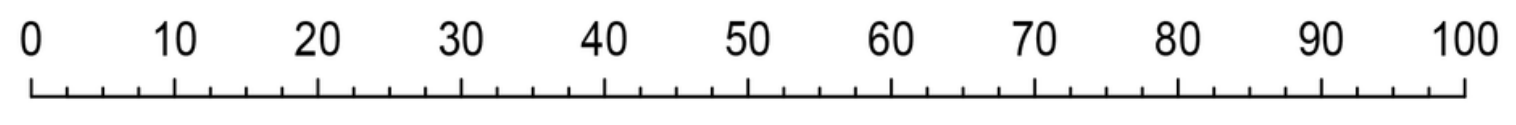

Grade

\section{Unknown}

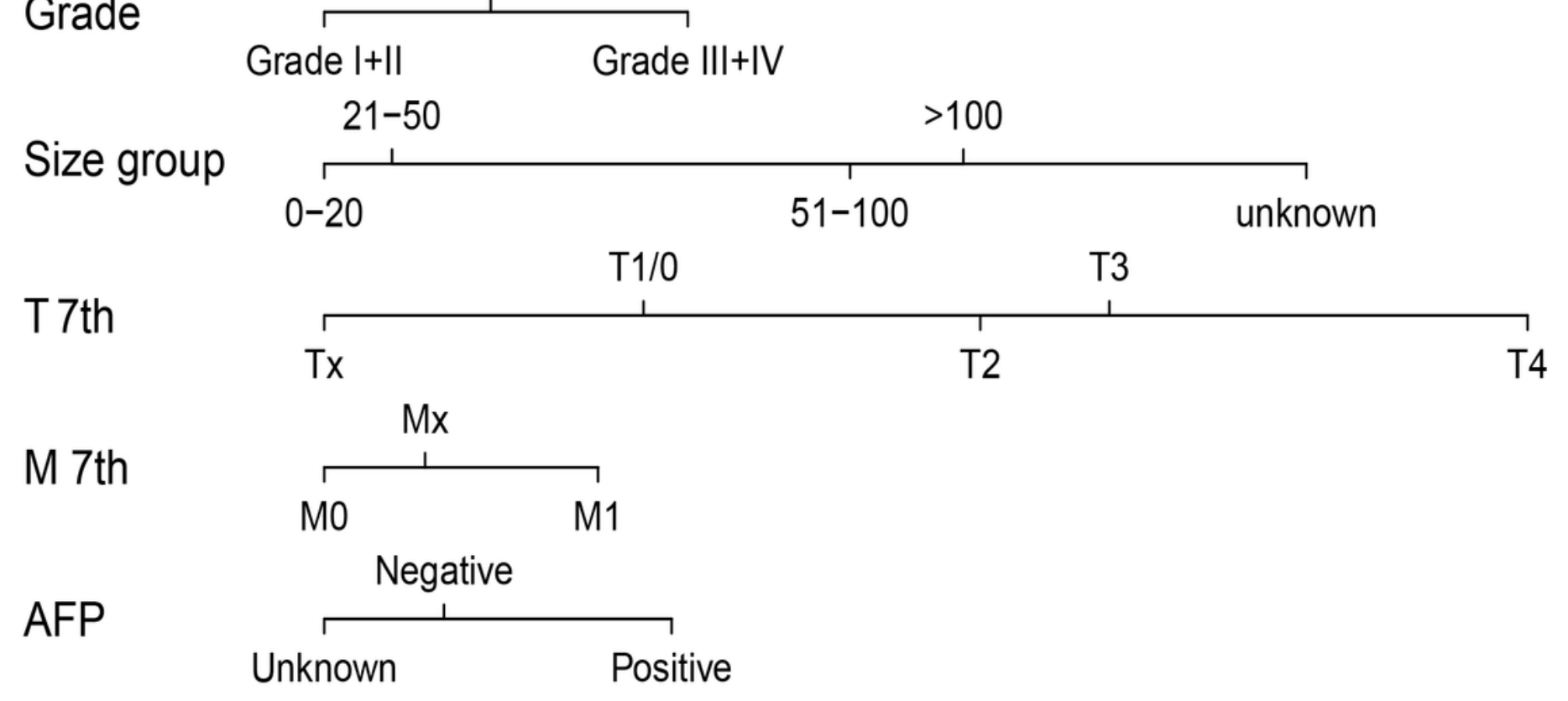

Total points

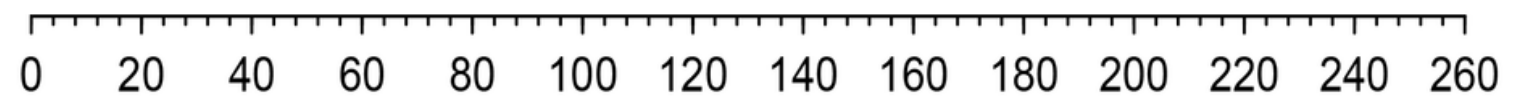

1-year Survival

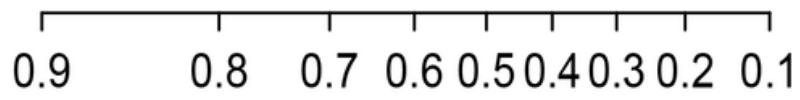

2-year Survival

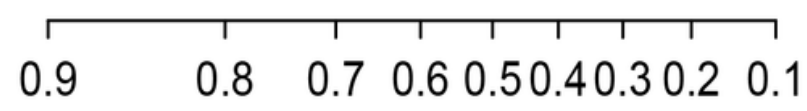

3-year Survival

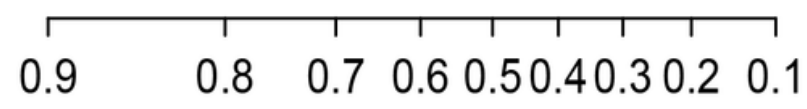

Figure 4

Nomogram predicting cancer specific-survival probability of young HCC patients after surgery 

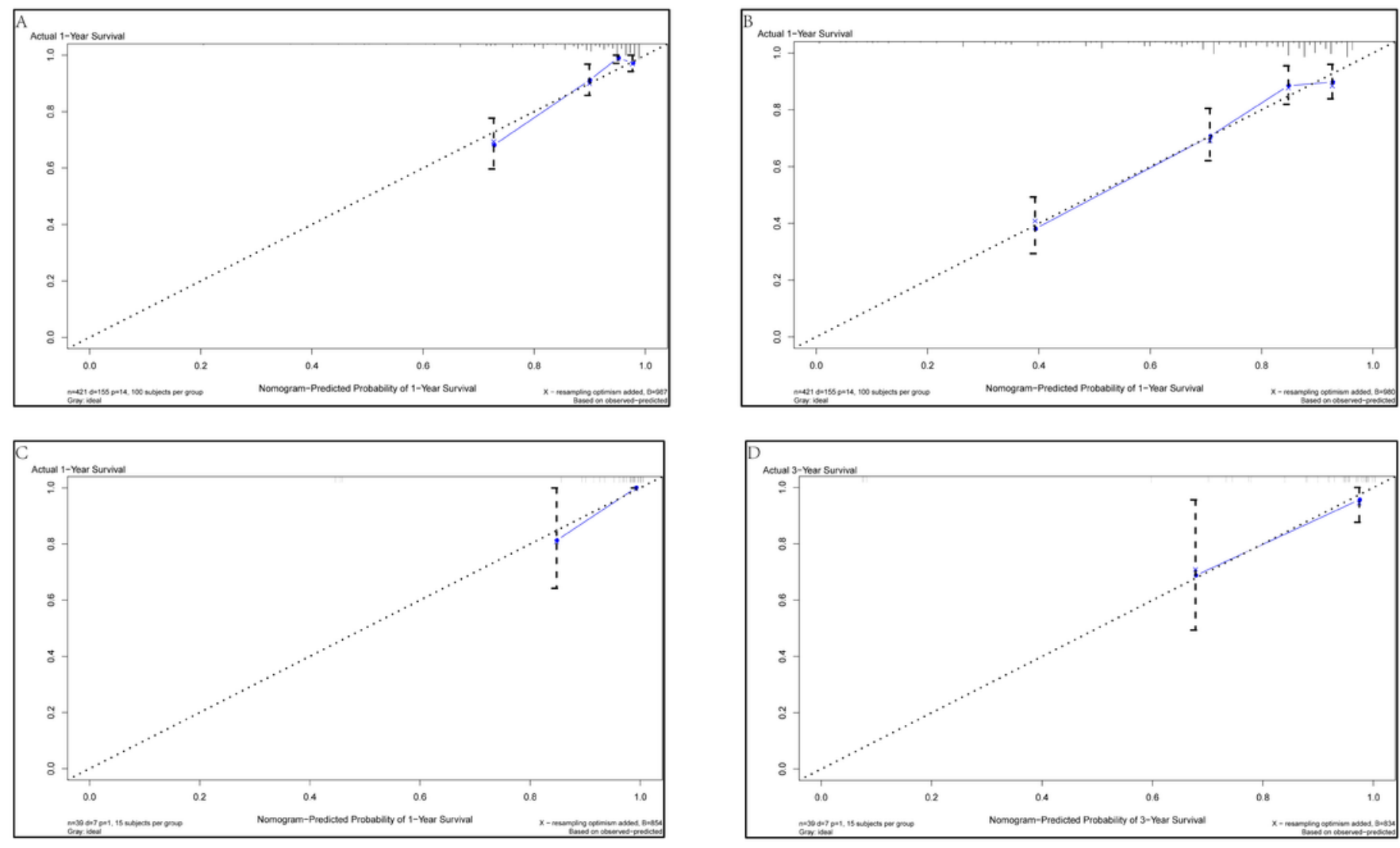

Figure 5

Calibration plot of the nomogram 5A. SEER training group nomogram-predicted probability of 1-year survival 5B. SEER training group nomogram-predicted probability of 3-year survival $5 \mathrm{C}$. CICAMS validation group nomogram-predicted probability of 1-year survival 5D. CICAMS validation group nomogrampredicted probability of 3-year survival 

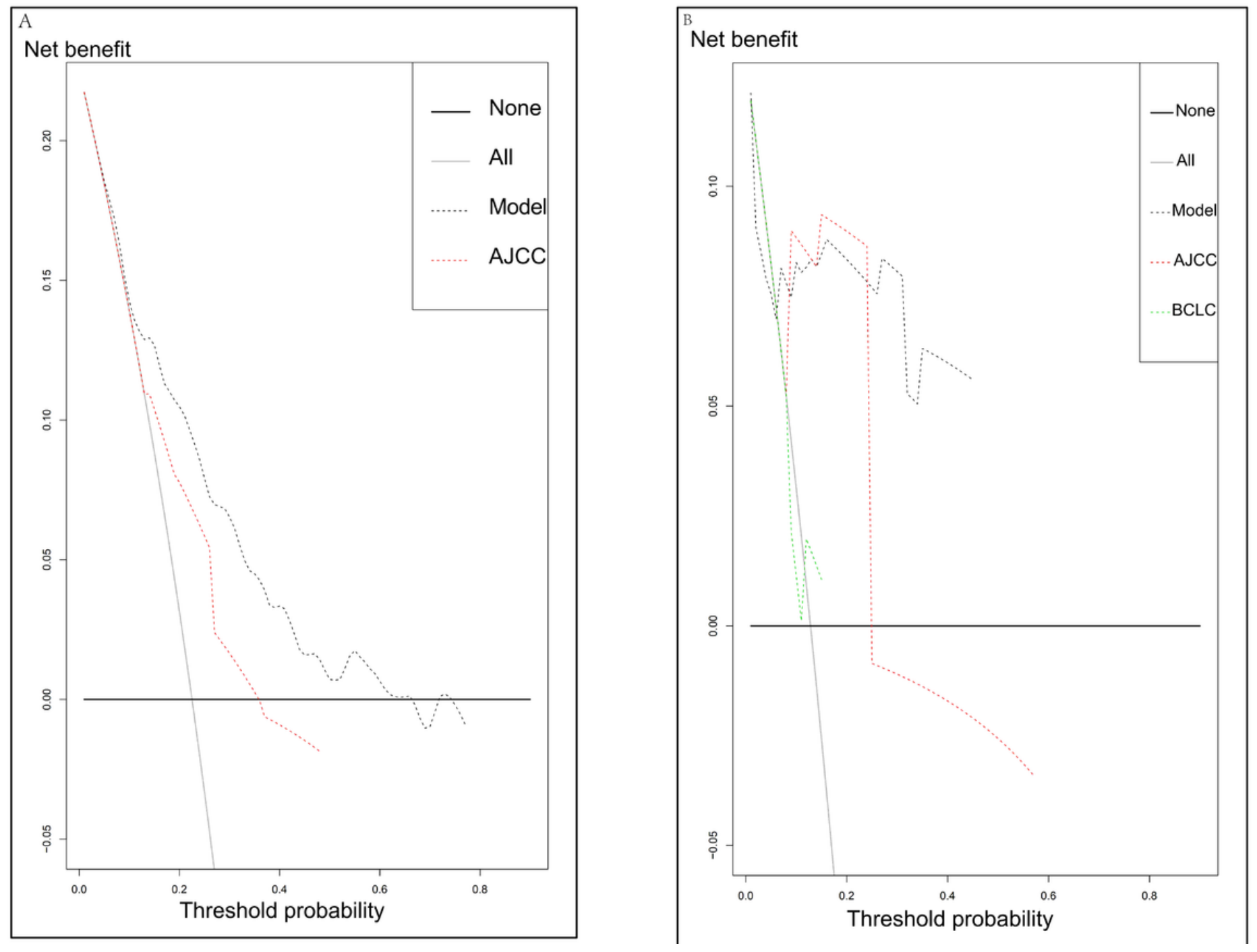

Figure 6

Decision curve analysis comparing the clinical utility of our nomogram model to the AJCC 7th staging system and BCLC staging system 6A. Decision curve analysis of training group 6B. Decision curve analysis of validation group 

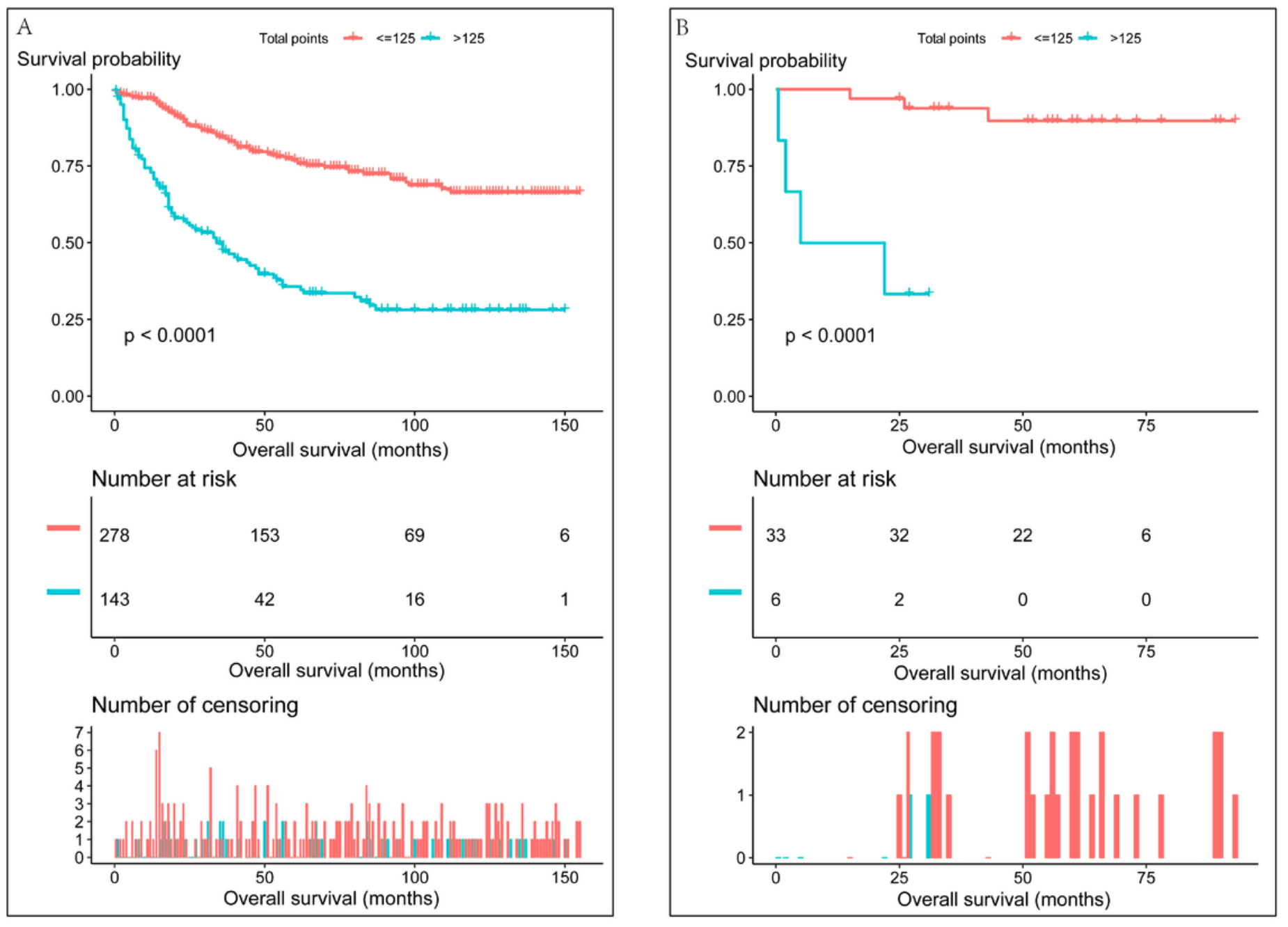

Figure 7

The survival curves of different risk groups according to the total points of 1257 A. Survival curves of training group 7B. Survival curves of validation group 